\title{
Elliptic, Yangian-Invariant "Leading Singularity"
}

\author{
Jacob L. Bourjaily $\odot,{ }^{1,2}$ Nikhil Kalyanapuram, ${ }^{1}$ Cameron Langer, ${ }^{1}$ Kokkimidis Patatoukos $\odot,{ }^{1}$ and Marcus Spradlin ${ }^{3,4}$ \\ ${ }^{1}$ Institute for Gravitation and the Cosmos, Department of Physics, Pennsylvania State University, \\ University Park, Pennsylvania 16802, USA \\ ${ }^{2}$ Niels Bohr International Academy and Discovery Center, Niels Bohr Institute, University of Copenhagen, \\ Blegdamsvej 17, DK-2100, Copenhagen Ø, Denmark \\ ${ }^{3}$ Department of Physics, Brown University, Providence, Rhode Island 02912, USA \\ ${ }^{4}$ Brown Theoretical Physics Center, Brown University, Providence, Rhode Island 02912, USA
}

(Received 1 January 2021; accepted 8 April 2021; published 17 May 2021)

\begin{abstract}
We derive closed formulas for the first examples of nonalgebraic, elliptic "leading singularities" in planar, maximally supersymmetric Yang-Mills theory and show that they are Yangian invariant.
\end{abstract}

DOI: 10.1103/PhysRevLett.126.201601

Introduction.-The residues of (the loop integrands of) scattering amplitudes on iterated, simple poles have played a critical and starring role through most of the incredible advances in our understanding of perturbative quantum field theory in recent years. Such residues, when they are of maximal codimension, have been called "leading singularities" [1,2] and have been used in the context of generalized unitarity [3-6] to construct integrands for amplitudes to impressive orders of perturbation [6-21]. Leading singularities have also appeared as the individual terms generated by Britto-Cachazo-Feng-Witten recursion relations for amplitudes at tree level [22,23] and beyond [24-26].

In the case of maximally supersymmetric Yang-Mills theory in the planar limit (SYM), leading singularities were discovered to enjoy an infinite-dimensional Yangian symmetry algebra [27-30] and were later found to be classified according to the "positroid" stratification of Grassmannian manifolds [31-33]. In Ref. [34], the correspondence between Yangian invariants and compact contour integrals over the positroid-canonical top form [35-37] in the Grassmannian was established.

As maximal codimension residues, leading singularities can be viewed as the coefficients of loop amplitude integrands that are locally (in loop-momentum space) " $d$ log" differential forms - wedge products of simple poles in all loop-momentum variables. (A multidimensional residue can in fact be defined by this fact alone-given by the inverse Jacobian of the requisite change of variables, evaluated at this point.) It is no surprise, therefore, that leading singularities also appear as the coefficients of polylogarithmic functions arising from "loop integration"

Published by the American Physical Society under the terms of the Creative Commons Attribution 4.0 International license. Further distribution of this work must maintain attribution to the author(s) and the published article's title, journal citation, and DOI. Funded by SCOAP . (over the Feynman contour of real momenta). Indeed, the connection between the sufficiency of leading singularities as information required to construct amplitudes and the existence of $d \log$ representations of loop integrands has been the subject of much research in recent years $[14,18,38,39]$.

Nevertheless, an increasing body of research has shown that many perturbative scattering amplitudes are not in fact polylogarithmic [40-43] but involve a much richer variety of functions - such as elliptic multiple polylogarithms [44-46] or even integrals over Calabi-Yau manifolds [47-50]. When the integrands of loop amplitudes are not $d \log$ somewhere locally, leading singularities as they have been so-far defined represent insufficient information (for reconstruction via unitarity) about perturbative amplitudes. The attempts to use generalized unitarity in such cases have relied on less intrinsically well-defined (or well-motivated) strategies-such as matching amplitude integrands functionally through some number of off-shell evaluations (see, e.g., $[16,18,51])$.

In this Letter, we argue that the notion of leading singularity must be broadened to include any fulldimensional compact, contour integral of an amplitude. Up to factors of $(2 \pi i)$, this definition automatically includes those functions covered previously but expands their scope to include contour integrals that do not just compute the residues around simple poles. We conjecture that this new, broadened definition of leading singularities represents complete unitarity-level information for amplitudes in perturbative SYM (and perhaps considerably beyond).

The first appearance of nonpolylogarithmic structure for planar SYM theory occurs in the ten-particle $\mathrm{N}^{3} \mathrm{MHV}$ amplitude at two loops [42]. In this Letter, we derive closed formulas for the elliptic leading singularities of this amplitude. We have checked that they are Yangian invariant. This check is very nontrivial since they involve the periods of complete elliptic integrals. Thus, they are not any 
elliptic analogs of "residues"-which would be necessarily algebraic — but rather contour integrals directly, carrying (some notion of) transcendental weight. Interestingly, the loop integrand whose coefficient would be an elliptic leading singularity takes the form of what has been defined as a "pure" elliptic integral [52,53]. We strongly suspect that this magic is not accidental: namely, that for any basis of loop integrands diagonalized on maximal-dimension, compact contours, the coefficients of amplitudes (in SYM) will be Yangian invariant and all integrands will be pure (in a sense suitably generalized to integrals involving higher-dimensional Calabi-Yau periods). But we leave such speculations to future work.

Elliptic subleading singularities of SYM.-We begin our analysis with the "double-box" cut of the two-loop, tenparticle $\mathrm{N}^{3} \mathrm{MHV}$ amplitude in planar SYM:

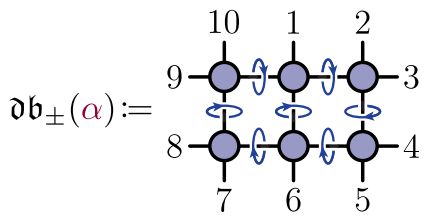

It is a "next-to-leading singularity" in the ordinary sense (a codimension-7 residue) and corresponds to a contour which encircles the seven poles from the propagators shown. This residue depends on one internal (on-shell) degree of freedom denoted $\alpha$ and a discrete label " \pm " signifying which of the two branches of the solution to the cut equations is taken. [As a positroid configuration, it corresponds to a 13-dimensional cell in $G_{+}(3,10)$ labeled by the decorated permutation $\{6,5,4,8,7,11,10,9,13,12\}$, integrated against the 12 constraints $\delta^{3 \times 4}(C \cdot Z)$-leaving a one-form over the remaining variable (see, e.g., [33,54]).]

We can give an explicit formula for the double-box cut $\mathfrak{D \mathfrak { b } _ { \pm }}(\alpha)$ as a single residue of (the relevant term of) the codimension-6 "kissing-triangle" function given in Ref. [16]:

$d \alpha d \beta \frac{1}{\alpha \beta} R[1, \hat{2}, \hat{6}, \hat{7}, \hat{1}] R[\hat{2}, 3,4,5,6] R[\hat{7}, 8,9,10,1]$,

where $R[a, b, c, d, e]$ is the $R$ invariant [55,56] with momentum supertwistor arguments $\left\{\mathcal{Z}_{a}, \ldots, \mathcal{Z}_{e}\right\}$ [57] and the "hatted" arguments are defined geometrically (see, e.g., [38]) by

$$
\begin{aligned}
& \hat{2}(\alpha):=\mathcal{Z}_{2}+\alpha \mathcal{Z}_{1}, \quad \hat{7}(\beta):=\mathcal{Z}_{7}+\beta \mathcal{Z}_{6}, \\
& \hat{6}(\alpha):=(56) \cap(\hat{2} 34), \quad \hat{1}(\beta):=(101) \cap(\hat{7} 89) .
\end{aligned}
$$

[Notice that the variables $\alpha$ and $\beta$ both carry nontrivial little-group weights. These could be absorbed by rescaling them (and the form) appropriately; but any such rescaling would cancel out of any complete compact contour integral (or residue).]
To obtain (1) from (2), we must compute the residue of (2) associated with the contour encircling the pole $\langle\hat{2} \hat{\sigma} \hat{7} \hat{1}\rangle=0$. We may take this residue with respect to either variable, and we choose to eliminate $\beta$. Because $\langle\hat{2} \hat{6} \hat{7} \hat{1}\rangle$ is quadratic in $\beta$, there are two branches available.

For the sake of concreteness, we note that

$$
\underset{\langle\hat{2} \hat{\operatorname{c} \hat{\jmath} \hat{1}\rangle=0}}{\operatorname{Res}}\left[\frac{d \beta}{\langle\hat{2} \hat{\sigma} \hat{\bar{\gamma}} \hat{1}\rangle}\right]= \pm \frac{c_{y}}{y(\alpha)},
$$

where $y(\alpha)^{2}$ is the (by-construction monic) quartic polynomial

$$
\begin{aligned}
\frac{1}{c_{y}^{2}} y^{2}(\alpha) & :=(\langle\hat{2} \hat{6} 6(789) \cap(101)\rangle+\langle\hat{2} \hat{6} 7(689) \cap(101)\rangle)^{2} \\
& -4\langle\hat{2} \hat{\sigma} 6(689) \cap(101)\rangle\langle\hat{2} \hat{\sigma} 7(789) \cap(101)\rangle,
\end{aligned}
$$

where $c_{y}^{2}$ is defined to be the inverse of the coefficient of $\alpha^{4}$ on the right-hand side of (5) [so as to render $y^{2}(\alpha)$ monic], and the particular solutions $\beta_{ \pm}^{*}$ to $\langle\hat{2} \hat{\sigma} \hat{7} \hat{1}\rangle=0$ are given by

$\beta_{ \pm}^{*}:=\frac{\langle\hat{2} \hat{\sigma} 6(789) \cap(101)\rangle+\langle\hat{2} \hat{\sigma} 7(689) \cap(101)\rangle \pm y(\alpha) / c_{y}}{2\langle\hat{2} \hat{\sigma}(689) \cap(101) 6\rangle}$.

To define the double-box residue (1), we may therefore replace the pole $\langle\hat{2} \hat{\sigma} \hat{7} \hat{1}\rangle$ (appearing in the denominator of $R[1, \hat{2}, \hat{6}, \hat{7}, \hat{1}])$ with $y(\alpha)$ and replace $\beta$ with $\beta_{ \pm}^{*}(\alpha)$ everywhere else in (2).

As $y^{2}(\alpha)$ is an irreducible quartic, the differential forms $\mathfrak{d} \mathfrak{b}_{ \pm}(\alpha)$ should be understood as involving the geometry of an elliptic curve. In general, any such differential form may be represented in the form

$$
\mathfrak{d} \mathfrak{b}_{ \pm}(\alpha)=: d \alpha\left[Q(\alpha) \pm \frac{1}{y(\alpha)} R(\alpha)\right]
$$

where $Q$ and $R$ are algebraic (super)functions of $\alpha$. As we are interested in taking a contour integral over the cycles of the elliptic curve, only the term involving $1 / y(\alpha)$ matters; we may extract this piece by writing

$$
\left[\mathfrak{d} \mathfrak{b}_{+}(\alpha)-\mathfrak{d} \mathfrak{b}_{-}(\alpha)\right]=: \mathfrak{d} \mathfrak{b}(\alpha)=: \frac{d \alpha}{y(\alpha)} \widehat{\mathfrak{d} \mathfrak{b}}(\alpha) .
$$

Analytic structure of the elliptic form $\mathfrak{d} \mathfrak{b}(\alpha)$.- - It is not hard to see that the differential form $\mathfrak{d b}(\alpha)$ has many simple poles-corresponding to the various factorization channels of the six four-particle amplitudes appearing in (1). Every such factorization channel has the topology of a "pentabox" leading singularity; counting every distinct solution to the cut equations for each topology, there are altogether 24 such "boundary" on-shell functions; let us denote them $\mathfrak{p b}_{i}$. 
Each of these "factorizations" of the double-box cut (1) corresponds to a simple pole located at $a_{i}$ in the $\alpha$ plane with residue equal to the corresponding pentabox on-shell function $\mathfrak{p b}_{i}$. For example, near

$$
\alpha \rightarrow a_{1}:=\frac{\langle 2(34) \cap(1012) 56\rangle}{\langle(34) \cap(1012) 561\rangle},
$$

the differential form $\mathfrak{d b}(\alpha)$ has a simple pole with residue

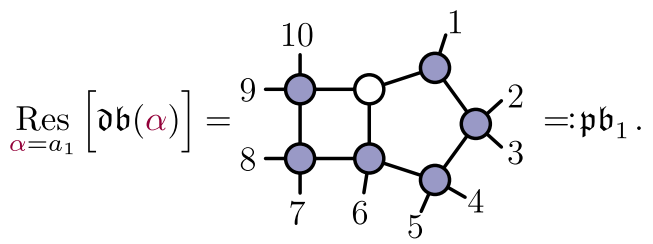

This function corresponds to one of the ordinary codimension-one boundaries of the positroid configuration of the double box; as such, it can easily be computed as the canonical (12-dimensional) form in the Grassmannian integrated against $\delta^{3 \times 4}(C \cdot Z)$. Regardless of how it is represented or computed, the location of each pole and its residue is easy to determine explicitly. Interestingly, it is worth noting that all of the poles $a_{i}$ lie on the real axis, and are negative semidefinite, for positive kinematics (see, e.g., [58] for a discussion of positive kinematics). These have been given explicitly in Supplemental Material [59].

Expanding $\mathfrak{d} \mathfrak{b}(\alpha)$ into a basis of forms with manifest simple poles results in a representation of $\delta \mathfrak{b}(\alpha)$ which may be written

$$
\mathfrak{d} \mathfrak{b}(\alpha)=: \frac{d \alpha}{y(\alpha)} \mathfrak{d} \mathfrak{b}_{0}+d \alpha \sum_{i=1}^{24} \frac{y\left(a_{i}\right)}{\left(\alpha-a_{i}\right) y(\alpha)} \mathfrak{p} \mathfrak{b}_{i},
$$

where $\mathfrak{d} \mathfrak{b}_{0}$ - the coefficient of the differential form $d \alpha / y(\alpha)$ without any simple poles-is therefore defined indirectly (but explicitly and without ambiguity) by

$$
\mathfrak{d \mathfrak { b } _ { 0 }}:=\widehat{\mathfrak{d b}}(\alpha)-\sum_{i=1}^{24} \frac{y\left(a_{i}\right)}{\left(\alpha-a_{i}\right)} \mathfrak{p} \mathfrak{b}_{i} .
$$

[Actually, for (10) and (11), there is in fact (exactly) one pole at $\alpha=-\infty$; for this term, the differential form in the sum should be understood as being $d \alpha[\alpha / y(\alpha)]$.]

Importantly, since $\delta \mathfrak{b}_{0}$ has no poles in $\alpha$ (including at infinity), it must be independent of $\alpha$-a fact that we have checked analytically. As such, it is worthwhile to express it in the form

$$
\mathfrak{d \mathfrak { b } _ { 0 }}:=\widehat{\mathfrak{d} \mathfrak{b}}\left(\alpha^{*}\right)-\sum_{i=1}^{24} \frac{y\left(a_{i}\right)}{\left(\alpha^{*}-a_{i}\right)} \mathfrak{p} \mathfrak{b}_{i}
$$

for any choice of $\alpha^{*}$. As every expression appearing in the right-hand side of (12) is fully known analytically [as superfunctions (or expressed in terms of $R$ invariants)], this provides a concrete definition for $\mathfrak{D} \mathfrak{b}_{0}$.

Elliptic leading singularities of SYM.-We are now in a position to determine the elliptic leading singularity-the integral of the form $\mathfrak{d b}(\alpha)$ over some choice of elliptic cycle, say, $\Omega_{a}$ :

$\mathfrak{e}_{a}:=\int_{\Omega_{a}} \mathfrak{d} \mathfrak{b}(\alpha)=\int_{\Omega_{a}} \frac{d \alpha}{y(\alpha)} \widehat{\mathfrak{d} \mathfrak{b}}(\alpha)= \pm 2 \int_{\Omega_{a}} \mathfrak{d} \mathfrak{b}_{ \pm}(\alpha)$.

To specify the particular elliptic cycle $\Omega_{a}$, it is worthwhile to note that, for positive (nondegenerate) momentumtwistor kinematics, it turns out that the roots $r_{i}$ of the quartic $y^{2}(\alpha)$,

$$
y^{2}(\alpha)=:\left(\alpha-r_{1}\right)\left(\alpha-r_{2}\right)\left(\alpha-r_{3}\right)\left(\alpha-r_{4}\right),
$$

always come in complex-conjugate pairs-between which we may introduce branch cuts. To be clear, we have chosen to order the roots such that $r_{1}^{*}=: r_{2}$ and $r_{3}^{*}=: r_{4}$, with $\operatorname{Re}\left(\mathrm{r}_{1}\right)>\operatorname{Re}\left(\mathrm{r}_{3}\right)$ and $\operatorname{Im}\left(\mathrm{r}_{1,3}\right)>0$; with this ordering of the roots (the reverse of the default ordering from Solve[] in Mathematica), the cross-ratio

$$
\phi:=\frac{\left(r_{2}-r_{1}\right)\left(r_{3}-r_{4}\right)}{\left(r_{2}-r_{3}\right)\left(r_{1}-r_{4}\right)}
$$

is always real; moreover, $\phi \in(0,1)$ for positive $\mathcal{Z}$ 's. With these conventions, we define $\Omega_{a}$ to be the contour "enclosing" the branch cut between the complex-conjugate pair of roots $r_{1,2}$ which does not encircle any of the simple poles of $\mathfrak{d} \mathfrak{b}(\alpha)$.

In order to compute the elliptic leading singularity (13), therefore, we merely need to note the basic period integrals appearing in (10):

$$
\int_{\Omega_{a}} d \alpha \frac{1}{y(\alpha)}=\frac{4 i}{\sqrt{\left(r_{3}-r_{2}\right)\left(r_{4}-r_{1}\right)}} K[\phi]
$$

and

$$
\begin{aligned}
& \int_{\Omega_{a}} d \alpha \frac{y\left(a_{i}\right)}{\left(\alpha-a_{i}\right) y(\alpha)}=\frac{4 i}{\sqrt{\left(r_{3}-r_{2}\right)\left(r_{4}-r_{1}\right)}}\left(\frac{y\left(a_{i}\right)}{\left(r_{4}-a_{i}\right)} K[\phi]\right. \\
& \left.+\frac{y\left(a_{i}\right)\left(r_{4}-r_{2}\right)}{\left(r_{2}-a_{i}\right)\left(r_{4}-a_{i}\right)} \Pi\left[\frac{\left(r_{4}-a_{i}\right)\left(r_{2}-r_{1}\right)}{\left(r_{2}-a_{i}\right)\left(r_{4}-r_{1}\right)}, \phi\right]\right),
\end{aligned}
$$

where $K[\phi]$ and $\Pi[q, \phi]$ are the complete elliptic integrals of the first and third kinds, respectively, defined in accordance with the conventions of Mathematica. Of particular importance is that both (16) and (17) are pure imaginary for positive kinematics; for the latter integral (17), this statement is nontrivial [even for $\phi \in(0,1)$ ], as the coefficients of both complete elliptic integrals appearing in (17) 
have nonzero real and imaginary parts and only the combination is pure imaginary. The full integral in (13) is obtained by integrating each term in (10) and using the explicit formula for $₫ \mathfrak{b}_{0}$ in (12) (for any choice of $\alpha^{*}$ ). As a consequence of the above discussion, this representation of $\mathfrak{e}_{a}$ is term-by-term purely imaginary.

One reason for our preference for the $a$ cycle (and also for our conventions regarding the roots) is that, in the space of positive kinematics, the only possible kinematic degenerations at codimension one result in the collision of one of the two pairs of complex-conjugate roots. When this happens, it is easy to see that both integrals (16) and (17) become equal to $(2 \pi i)$ times the residue around the corresponding simple pole generated by the colliding pair of roots. (Recall that $K[0]=\Pi[0,0]=\pi / 2$.) The $b$-cycle integrals, in contrast, diverge upon such degenerations.

More concise formulas for the leading singularities.-In the discussion above, the reader should notice that every pentabox on-shell function $\mathfrak{p b}_{i}$ appears twice: once in the definition of $\mathfrak{d b}_{0}$ in (12) (where $\alpha^{*}$ may be taken a $s$ arbitrary) and once as the coefficient of the particular ( $\alpha$-dependent) differential form in $\mathfrak{d b}(\alpha)$ in (10). From the first, (16) results in a contribution to $\mathfrak{e}_{a}$ of

$\mathfrak{e}_{a} \supset-\frac{4 i}{\sqrt{\left(r_{3}-r_{2}\right)\left(r_{4}-r_{1}\right)}} K[\phi] \times \frac{y\left(a_{i}\right)}{\left(\alpha^{*}-a_{i}\right)} \mathfrak{p} \mathfrak{b}_{i} ;$

from the second, (17) results in a contribution of

$$
\begin{aligned}
\mathfrak{e}_{a} \supset & \frac{4 i}{\sqrt{\left(r_{3}-r_{2}\right)\left(r_{4}-r_{1}\right)}}\left(\frac{y\left(a_{i}\right)}{\left(r_{4}-a_{i}\right)} K[\phi]\right. \\
& \left.+\frac{y\left(a_{i}\right)\left(r_{4}-r_{2}\right)}{\left(r_{2}-a_{i}\right)\left(r_{4}-a_{i}\right)} \Pi\left[\frac{\left(r_{4}-a_{i}\right)\left(r_{2}-r_{1}\right)}{\left(r_{2}-a_{i}\right)\left(r_{4}-r_{1}\right)}, \phi\right]\right) \mathfrak{p} \mathfrak{b}_{i} .
\end{aligned}
$$

From these two contributions, mere pattern recognition suggests a "preferential" choice for the arbitrary point $\alpha^{*}$. In particular, if we were to take $\alpha^{*}$ to be $r_{4}$, all the terms involving $K[\phi] \times \mathfrak{p b}_{i}$ would cancel. As $\alpha^{*}$ is indeed arbitrary, this would result in a final, more compact expression:

$$
\begin{aligned}
\mathfrak{e}_{a}= & \frac{4 i}{\sqrt{\left(r_{3}-r_{2}\right)\left(r_{4}-r_{1}\right)}}\left(K[\phi] \widehat{\mathfrak{d} \mathfrak{b}}\left(\alpha^{*} \rightarrow r_{4}\right)\right. \\
& \left.+\sum_{i=1}^{24} \frac{y\left(a_{i}\right)\left(r_{4}-r_{2}\right)}{\left(r_{2}-a_{i}\right)\left(r_{4}-a_{i}\right)} \Pi\left[\frac{\left(r_{4}-a_{i}\right)\left(r_{2}-r_{1}\right)}{\left(r_{2}-a_{i}\right)\left(r_{4}-r_{1}\right)}, \phi\right] \mathfrak{p} \mathfrak{b}_{i}\right) .
\end{aligned}
$$

The reader may be worried about the fact that taking $\alpha^{*}$ to be $r_{4}$ sets $y\left(\alpha^{*}\right) \rightarrow 0$. As such, the first term in (20) may appear ill defined. However, $y\left(\alpha^{*}\right)$ also appears (manifestly) in the denominator in the definition of the differential form $\mathfrak{d b}\left(\alpha^{*}\right)$; as such, the evaluation-for $\widehat{\mathfrak{d b}}(\alpha)$-may be performed without taking limits (and turns out to be extremely stable, numerically). This simplified form is included in Supplemental Material [59].

It is worth noting that, upon any physical degeneration (at codimension one), the elliptic function $\mathfrak{e}_{a}$ in fact vanishes identically. This can be understood by noting that any such physical degeneration would correspond to an ordinary "residue" contour about the simple pole generated by the collision of the roots- $[(2 \pi i)$ times the residue $]$ about the point $\alpha=r_{1}$ or $r_{4}$; as there is no corresponding on-shell function to draw, the amplitude must vanish on such a contour. We have checked that it does.

The $b$-cycle elliptic leading singularity-the one encircling a branch cut between the roots $r_{1}$ and $r_{3}$, say (and not encircling any of the simple poles) — is easily obtained from our work above [replacing $r_{2} \leftrightarrow r_{3}$ in (16) and (17)]. Using these expressions for the $b$-cycle integrals of the relevant differential forms and choosing $\alpha^{*}$ to be $r_{4}$ as before, the resulting expression becomes

$$
\begin{aligned}
\mathfrak{e}_{b}= & \frac{4}{\sqrt{\left(r_{3}-r_{2}\right)\left(r_{4}-r_{1}\right)}}\left(K[1-\phi] \widehat{\mathfrak{d} \mathfrak{b}}\left(\alpha^{*} \rightarrow r_{4}\right)\right. \\
& \left.+\sum_{i=1}^{24} \frac{y\left(a_{i}\right)\left(r_{4}-r_{3}\right)}{\left(r_{3}-a_{i}\right)\left(r_{4}-a_{i}\right)} \Pi\left[\frac{\left(r_{4}-a_{i}\right)\left(r_{3}-r_{1}\right)}{\left(r_{3}-a_{i}\right)\left(r_{4}-r_{1}\right)}, 1-\phi\right] \mathfrak{p b}_{i}\right) .
\end{aligned}
$$

One interesting aspect of these formulas is that the $b$-cycle leading singularity $\mathfrak{e}_{b}$ is invariant under a fivefold cyclic rotation (in a highly nontrivial way), while the $a$-cycle integral $\mathfrak{e}_{a}$ is not-reflecting the asymmetry of the contour (analogous to the noncyclic invariance of the four-mass box leading singularities).

Explicit, computer-usable (Mathematica) expressions for both elliptic leading singularities $\mathfrak{e}_{a}$ and $\mathfrak{e}_{b}$ are included as Supplemental Material [59]. This code makes use of tools made available in Refs. [14,16,60].

Yangian invariance of the elliptic leading singularities.Among the most interesting aspects of our results so far is that Yangian invariance requires the complete elliptic integrals $K[\phi]$ and $\Pi[q, \phi]$ as coefficients appearing in their definition. The easiest way to see this is to consider one of the level-one generators (see, e.g., [30]):

$$
J_{B}^{A}:=\sum_{a=1}^{n} \mathcal{Z}_{a}^{A} \frac{\partial}{\partial \mathcal{Z}_{a}^{B}}
$$

where $\mathcal{Z}_{a}$ is a supermomentum twistor and the component $A$ is taken to be fermionic and $B$ bosonic. This operator turns out to be surprisingly powerful. For example, it tells us that any nontrivial function of cross-ratios times a Yangian invariant will not be Yangian invariant. In particular, direct application of this operator demonstrates that 
the four-mass box coefficient as defined in Ref. [14], which is not simply a product of $R$ invariants but includes as part of its definition a particular function of the relevant crossratios, is only Yangian invariant with these peculiar prefactors included.

It turns out that no combination or subset of the terms (with constant coefficients) that appear in the two formulas for $\mathfrak{e}_{a, b}$ in (20) and (21), respectively, is Yangian invariant except for the $\mathfrak{e}_{a, b}$ themselves. We have checked this explicitly using numerical approximations for the derivatives appearing in the Yangian generator (22).

That any integral over a compact, full-dimensional cycle in the Grassmannian should be Yangian invariant may not be surprising: indeed, it seems to be a consequence of the arguments described in Ref. [34]. However, the fact that these integrals, in the case of elliptic contours, require the nonalgebraic content of complete elliptic integrals is very surprising, as this is in stark contrast with the notion of taking residues (a purely algebraic operation).

The lesson here has very obvious consequences for generalization beyond elliptic contours-which we must leave to future work.

Conclusions and discussion.-In this Letter, we have motivated a broader definition of leading singularity to be any full-dimensional compact, contour integral of a perturbative amplitude's loop integrand. This definition differs from the previous one merely by factors of $(2 \pi i)$ in the case of simple (logarithmic) poles but includes also the contour integrals of elliptic curves. We have given closed formulas for elliptic-containing contour integrals for the first nonpolylogarithmic structure that arises in planar SYM theory, and we have checked that they are Yangian invariant. We conjecture that, with this broader definition, all leading singularities of SYM will be Yangian invariant and that the set of all leading singularities will represent complete information for perturbative amplitudes in this theory. We will have more to say about this in a forthcoming work.

The authors gratefully acknowledge fruitful conversations with Lauren Altman, Nima Arkani-Hamed, Claude Duhr, Song He, Enrico Herrmann, Julio Parra-Martinez, Cristian Vergu, and Anastasia Volovich. This work was performed in part at the Aspen Center for Physics, which is supported by National Science Foundation Grant No. PHY1607611, and the Harvard Center of Mathematical Sciences and Applications. This project has been supported by an ERC Starting Grant (No. 757978), a grant from the Villum Fonden (No. 15369), and a grant from the Simons Foundation (341344, LA) (J. L. B.) and was supported in part by the U.S. Department of Energy under Contract No. DE-SC0010010 Task A (M. S.).

[1] R. J. Eden, P. V. Landshoff, D. I. Olive, and J. C. Polkinghorne, The Analytic S-Matrix (Cambridge University Press, Cambridge, England, 1966).
[2] F. Cachazo, Sharpening the leading singularity, arXiv: 0803.1988.

[3] Z. Bern, L. J. Dixon, D. C. Dunbar, and D. A. Kosower, One-loop $n$-point gauge theory amplitudes, unitarity and collinear limits, Nucl. Phys. B425, 217 (1994).

[4] Z. Bern, L. J. Dixon, D. C. Dunbar, and D. A. Kosower, Fusing gauge theory tree amplitudes into loop amplitudes, Nucl. Phys. B435, 59 (1995).

[5] Z. Bern and A. G. Morgan, Massive loop amplitudes from unitarity, Nucl. Phys. B467, 479 (1996).

[6] R. Britto, F. Cachazo, and B. Feng, Generalized unitarity and one-loop amplitudes in $\mathcal{N}=4$ super-Yang-Mills, Nucl. Phys. B725, 275 (2005).

[7] E. I. Buchbinder and F. Cachazo, Two-loop amplitudes of gluons and octa-cuts in $\mathcal{N}=4$ super Yang-Mills, J. High Energy Phys. 11 (2005) 036.

[8] Z. Bern, M. Czakon, L. J. Dixon, D. A. Kosower, and V. A. Smirnov, The four-loop planar amplitude and cusp anomalous dimension in maximally supersymmetric Yang-Mills theory, Phys. Rev. D 75 (2007) 085010.

[9] Z. Bern, J. J. M. Carrasco, H. Johansson, and D. A. Kosower, Maximally supersymmetric planar yang-mills amplitudes at five loops, Phys. Rev. D 76, 125020 (2007).

[10] F. Cachazo and D. Skinner, On the structure of scattering amplitudes in $\mathcal{N}=4$ super Yang-Mills and $\mathcal{N}=8$ supergravity, arXiv:0801.4574.

[11] F. Cachazo, M. Spradlin, and A. Volovich, Leading singularities of the two-loop six-particle MHV amplitude, Phys. Rev. D 78, 105022 (2008).

[12] M. Spradlin, A. Volovich, and C. Wen, Three-loop leading singularities and BDS ansatz for five particles, Phys. Rev. D 78, 085025 (2008).

[13] J. L. Bourjaily, A. DiRe, A. Shaikh, M. Spradlin, and A. Volovich, The soft-collinear bootstrap: $\mathcal{N}=4$ Yang-Mills amplitudes at six and seven loops, J. High Energy Phys. 03 (2012) 032.

[14] J. L. Bourjaily, S. Caron-Huot, and J. Trnka, Dualconformal regularization of infrared loop divergences and the chiral box expansion, J. High Energy Phys. 01 (2015) 001.

[15] J. L. Bourjaily, P. Heslop, and V.-V. Tran, Perturbation Theory at Eight Loops: Novel Structures and the Breakdown of Manifest Conformality in $\mathcal{N}=4$ Supersymmetric Yang-Mills Theory, Phys. Rev. Lett. 116, 191602 (2016).

[16] J. L. Bourjaily and J. Trnka, Local integrand representations of all two-loop amplitudes in planar SYM, J. High Energy Phys. 08 (2015) 119.

[17] J. L. Bourjaily, P. Heslop, and V.-V. Tran, Amplitudes and correlators to ten loops using simple, graphical bootstraps, J. High Energy Phys. 11 (2016) 125.

[18] J.L. Bourjaily, E. Herrmann, and J. Trnka, Prescriptive unitarity, J. High Energy Phys. 06 (2017) 059.

[19] J. L. Bourjaily, E. Herrmann, and J. Trnka, Maximally supersymmetric amplitudes at infinite loop momentum, Phys. Rev. D 99, 066006 (2019).

[20] J. L. Bourjaily, E. Herrmann, C. Langer, A. J. McLeod, and J. Trnka, All-Multiplicity Nonplanar Amplitude Integrands in Maximally Supersymmetric Yang-Mills Theory at Two Loops, Phys. Rev. Lett. 124, 111603 (2020).

[21] J. L. Bourjaily, E. Herrmann, C. Langer, A. J. McLeod, and J. Trnka, Prescriptive unitarity for non-planar six-particle 
amplitudes at two loops, J. High Energy Phys. 12 (2019) 073.

[22] R. Britto, F. Cachazo, and B. Feng, New recursion relations for tree amplitudes of gluons, Nucl. Phys. B715, 499 (2005).

[23] R. Britto, F. Cachazo, B. Feng, and E. Witten, Direct Proof of Tree-Level Recursion Relation in Yang- Mills Theory, Phys. Rev. Lett. 94, 181602 (2005).

[24] S. Caron-Huot, Loops and trees, J. High Energy Phys. 05 (2011) 080.

[25] N. Arkani-Hamed, J. L. Bourjaily, F. Cachazo, S. CaronHuot, and J. Trnka, The all-loop integrand for scattering amplitudes in planar $\mathcal{N}=4$ SYM, J. High Energy Phys. 01 (2011) 041.

[26] R. H. Boels and R. S. Isermann, New relations for scattering amplitudes in yang-mills theory at loop level, Phys. Rev. D 85, 021701(R) (2012).

[27] J. Drummond, J. Henn, G. Korchemsky, and E. Sokatchev, Dual superconformal symmetry of scattering amplitudes in $\mathcal{N}=4$ super Yang-Mills theory, Nucl. Phys. B828, 317 (2010).

[28] L. F. Alday and R. Roiban, Scattering amplitudes, Wilson loops and the string/gauge theory correspondence, Phys. Rep. 468, 153 (2008).

[29] J. M. Drummond, J. M. Henn, and J. Plefka, Yangian Symmetry of scattering amplitudes in $\mathcal{N}=4$ super YangMills theory, J. High Energy Phys. 05 (2009) 046.

[30] J. Drummond and L. Ferro, Yangians, Grassmannians and T-duality, J. High Energy Phys. 07 (2010) 027.

[31] A. Postnikov, Total positivity, Grassmannians, and networks, arXiv:math/0609764.

[32] L. K. Williams, Enumeration of totally positive Grassmann cells, Adv. Math. 190, 319 (2005).

[33] N. Arkani-Hamed, J. L. Bourjaily, F. Cachazo, A. B. Goncharov, A. Postnikov, and J. Trnka, Grassmannian Geometry of Scattering Amplitudes (Cambridge University Press, Cambridge, England, 2016).

[34] J. Drummond and L. Ferro, The Yangian origin of the Grassmannian integral, J. High Energy Phys. 12 (2010) 010.

[35] N. Arkani-Hamed, F. Cachazo, C. Cheung, and J. Kaplan, A duality for the $S$-matrix, J. High Energy Phys. 03 (2010) 020 .

[36] N. Arkani-Hamed, F. Cachazo, and C. Cheung, The Grassmannian origin of dual superconformal invariance, J. High Energy Phys. 03 (2010) 036.

[37] L. Mason and D. Skinner, Dual superconformal invariance, momentum twistors and Grassmannians, J. High Energy Phys. 11 (2009) 045.

[38] N. Arkani-Hamed, J. L. Bourjaily, F. Cachazo, and J. Trnka, Local integrals for planar scattering amplitudes, J. High Energy Phys. 06 (2012) 125.

[39] N. Arkani-Hamed, J. L. Bourjaily, F. Cachazo, and J. Trnka, Singularity Structure of Maximally Supersymmetric Scattering Amplitudes, Phys. Rev. Lett. 113, 261603 (2014).

[40] D. J. Broadhurst, J. Fleischer, and O. V. Tarasov, Two loop two-point functions with masses: Asymptotic expansions and taylor series, in any dimension, Z. Phys. C 60, 287 (1993).
[41] S. Bloch and P. Vanhove, The elliptic dilogarithm for the sunset graph, J. Number Theory 148, 328 (2015).

[42] S. Caron-Huot and K. J. Larsen, Uniqueness of two-loop master contours, J. High Energy Phys. 10 (2012) 026.

[43] J. L. Bourjaily, A. J. McLeod, M. Spradlin, M. von Hippel, and M. Wilhelm, Elliptic Double-Box Integrals: Massless Scattering Amplitudes beyond Polylogarithms, Phys. Rev. Lett. 120, 121603 (2018).

[44] E. Remiddi and L. Tancredi, An elliptic generalization of multiple polylogarithms, Nucl. Phys. B925, 212 (2017).

[45] J. Brödel, C. Duhr, F. Dulat, and L. Tancredi, Elliptic polylogarithms and iterated integrals on elliptic curves i: General formalism, J. High Energy Phys. 05 (2018) 093.

[46] J. Brödel, C. Duhr, F. Dulat, and L. Tancredi, Elliptic polylogarithms and iterated integrals on elliptic curves II: An application to the sunrise integral, Phys. Rev. D 97, 116009 (2018).

[47] F. Brown and O. Schnetz, A K3 in $\phi^{4}$, Duke Math. J. 161, 1817 (2012).

[48] J. L. Bourjaily, Y.-H. He, A. J. McLeod, M. von Hippel, and M. Wilhelm, Traintracks Through Calabi-Yaus: Amplitudes Beyond Elliptic Polylogarithms, Phys. Rev. Lett. 121, 071603 (2018).

[49] J.L. Bourjaily, A. J. McLeod, M. von Hippel, and M. Wilhelm, Bounded Collection of Feynman Integral Calabi-Yau Geometries, Phys. Rev. Lett. 122, 031601 (2019).

[50] J. L. Bourjaily, A. J. McLeod, C. Vergu, M. Volk, M. Von Hippel, and M. Wilhelm, Embedding Feynman integral (Calabi-Yau) geometries in weighted projective space, J. High Energy Phys. 01 (2020) 078.

[51] J. L. Bourjaily, E. Herrmann, C. Langer, and J. Trnka, Building bases of loop integrands, J. High Energy Phys. 11 (2020) 116.

[52] J. Bröedel, C. Duhr, F. Dulat, B. Penante, and L. Tancredi, Elliptic Feynman integrals and pure functions, J. High Energy Phys. 01 (2019) 023.

[53] A. Primo and L. Tancredi, Maximal cuts and differential equations for Feynman integrals. An application to the three-loop massive banana graph, Nucl. Phys. B921, 316 (2017).

[54] J. L. Bourjaily, Positroids, Plabic graphs, and scattering amplitudes in Mathematica, arXiv:1212.6974.

[55] J. M. Drummond and J. M. Henn, All tree-level amplitudes in $\mathcal{N}=4$ SYM, J. High Energy Phys. 04 (2009) 018.

[56] M. Bullimore, L. Mason, and D. Skinner, MHV diagrams in momentum twistor space, J. High Energy Phys. 12 (2010) 032.

[57] A. Hodges, Eliminating spurious poles from gauge-theoretic amplitudes, J. High Energy Phys. 05 (2013) 135.

[58] N. Arkani-Hamed and J. Trnka, The amplituhedron, J. High Energy Phys. 10 (2014) 30.

[59] See Supplemental Material at http://link.aps.org/supplemental/ 10.1103/PhysRevLett.126.201601 for Mathematica notebook implementing the elliptic leading singularities $\mathfrak{e}_{a}$ and $\mathfrak{e}_{b}$.

[60] J. L. Bourjaily, Efficient tree-amplitudes in $\mathcal{N}=4$ : Automatic BCFW recursion in Mathematica, arXiv:1011. 2447. 